\title{
Association of endothelial progenitor cells and peptic ulcer treatment in patients with type $\mathbf{2}$ diabetes mellitus
}

\author{
ZHIHONG NIE ${ }^{1}$, LIMIN XU ${ }^{2}$, CHUANYUAN LI $^{1}$, TAO TIAN ${ }^{1}$, PINGPING XIE ${ }^{1}$, XIA CHEN ${ }^{1}$ and BOJING LI ${ }^{1}$ \\ Departments of ${ }^{1}$ Gastroenterology, and ${ }^{2}$ Laboratory Medicine, Gongli Hospital, \\ The Second Military Medicine University, Shanghai 200135, P.R. China
}

Received November 12, 2014; Accepted December 17, 2015

DOI: $10.3892 /$ etm.2016.3114

\begin{abstract}
The present study aimed to investigate the association between endothelial progenitor cells (EPCs) and peptic ulcers in patients with or without type 2 diabetes mellitus (T2DM), in association with the efficiency of peptic ulcer treatment. The study recruited healthy subjects and peptic ulcer patients with or without T2DM. All the ulcer patients, including those with and without T2DM, were administered omeprazole for 8 weeks. Peptic ulcer patients with T2DM were additionally treated with glipizide and novolin. Blood samples were then obtained from the three groups following ulcer treatment. CD133+ cells were isolated from the blood samples using magnetic bead selection, and cultured in complete medium 199. Morphological and quantity changes in EPCs were observed by light and fluorescence microscopy. In addition, flow cytometric analysis was used to quantify the number of vascular endothelial cells. The treatment was partially effective in 7 of the 32 peptic ulcer patients without T2DM and 12 of the 32 peptic ulcer patients with T2DM. However, this treatment was ineffective in 20 of the 32 peptic ulcer patients with T2DM. Notably, 25 peptic ulcer patients without T2DM were defined as completely recovered following treatment. In addition, the number of circulating EPCs as well as their colony forming ability was significantly reduced $(\mathrm{P}<0.05)$ in the peptic ulcer patients with T2DM following ulcer treatment, compared with the other groups. Circulating EPC counts were significantly increased in peptic ulcer patients without T2DM, as compared with the healthy controls. With regards to colony formation, peptic ulcer patients without T2DM did not exhibit improved colony formation ability. In conclusion, the number of circulating EPCs and their colony-forming ability was significantly reduced in peptic ulcer patients with T2DM following ulcer treatment when compared with the other groups. This suggests
\end{abstract}

Correspondence to: Mr. Bojing Li, Department of Gastroenterology, Gongli Hospital, The Second Military Medicine University, 219 Miaopu Road, Pudong New Area, Shanghai 200135, P.R. China E-mail: bojinglibjl@163.com

Key words: peptic ulcer, type 2 diabetes mellitus, endothelial progenitor cells, curative effect that the poor curative effect of peptic ulcer treatment in these patients is associated with impairment of EPCs.

\section{Introduction}

Peptic ulcer development is one of the most frequent complications of type 2 diabetes mellitus (T2DM) due to the increased likelihood of Helicobacter pylori (H. pylori) infection in T2DM patients, resulting in symptoms such as bleeding and perforation (1). The symptoms of peptic ulcers also include a burning sensation, belching, weight loss and poor appetite. Peptic ulcers affect $4 \%$ of the population and were the cause of 301,000 deaths in 2013 (2,3). T2DM is a metabolic disorder characterized by hyperglycemia in association with insulin resistance and lack of insulin (4). Furthermore, ulcers are the most common complication of metabolic disorders, and are associated with severe pathological lesions, such as extensive vascular lesions, and mucosa ischemic necrosis (5).

Endothelial progenitor cells (EPCs) are biological markers for vascular function, since they have an important role in vascular repair and angiogenesis (6). The development of T2DM has been demonstrated to be closely associated with low levels of circulating EPCs (7). Furthermore, a previous study has reported that peripheral vascular disease in T2DM patients was associated with a low number of EPCs (8). Although the role of EPCs in ulcer healing in humans has yet to be investigated, a reduction of EPCs in patients with diabetic foot ulcers has been demonstrated (9). Therefore, the hypothesis that EPC injury is associated with T2DM and contributes to a poor clinical outcome in peptic ulcer patients with T2DM requires further investigation.

In the present study, circulating EPCs were obtained from the blood samples of three groups, including peptic ulcer patients with T2DM, peptic ulcer patients without T2DM and healthy controls. The study aimed to examine the association of the quantity and function of circulating EPCs with the curative effect of various treatments, in order to provide novel strategies for the treatment of peptic ulcers in patients with T2DM.

\section{Patients and methods}

Patients and groups. All subjects were recruited from the Department of Gastroenterology, Gongli Hospital (Shanghai, China) between January 2011 and December 2013. In total, 
three groups of patients were examined: Peptic ulcer patients with T2DM (group A; $n=32$; age, $64.4 \pm 6.3$ years; 18 male and 14 female); peptic ulcer patients without T2DM (group B; $n=32$; age, $65.1 \pm 5.8$ years; 17 male and 15 female); and healthy control subjects (group C; $n=32$; age, $64.8 \pm 6.9$ years; 18 male and 14 female).

Prior to inclusion into the present study, subjects underwent T2DM evaluation using the diagnostic criteria for DM as determined by the World Health Organization (10), and peptic ulcer disease was evaluated using the assessment of diagnosis of peptic ulcer reviewed by the Editorial Board of Chinese Journal of Digestion (11). Furthermore, an endoscopy and $H$. pylori infection diagnosis were conducted to further determine the health conditions of the patients. The healthy control patients were also subjected to blood glucose examination, the $14 \mathrm{C}$-urea breath test and gastric biopsies.

Subjects with the following characteristics were excluded from the study: i) Malignant lesions in the gastric ulcers, which were identified using pathology techniques; ii) concurrent severe $H$. pylori infection and acidosis in the patients with T2DM; iii) severe complications associated with the ulcers; iv) drug administration, such as non-steroidal anti-inflammatory drugs, corticosteroids or statins; v) acute myocardial infarction, angina and peripheral vascular disease; or vi) having undergone surgery of any kind within the last 24 months.

The present study was approved by the Ethics Committee of Gongli Hospital and written informed consent was obtained from all participants.

Treatment protocols. Peptic ulcer patients with T2DM were treated with $10 \mathrm{mg}$ glipizide daily (Pfizer, Inc., New York, NY, USA) to lower the blood glucose levels, and patients with characteristics of hematemesis or hematochezia were treated with daily injections of 8 units novolin (Novo Nordisk, Bagsvaerd, Denmark). Omeprazole (20 mg; Hainan Haili Pharmaceutical Co. Ltd., Haikou, China) was administered as an antiulcer proton pump inhibitor for 8 weeks in peptic ulcer patients with or without T2DM. In addition, H. pylori infection in peptic ulcer patients was treated with a combination of amoxicillin ( $0.5 \mathrm{~g}$ every $8 \mathrm{~h}$; CSPC Pharmaceutical Group, Shijiazhuang, China), clarithromycin (250 mg every $12 \mathrm{~h}$; Abbott Laboratories, Lake Bluff, IL, USA) and metronidazole (1.2 g daily; Novartis, Basel, Switzerland) for 2 weeks. This treatment regimen was maintained for 8 weeks.

Evaluation of treatment effect. The curative effects of the treatments were evaluated based on clinical symptoms and endoscopy results. Various scales of treatment efficacy were defined: i) Complete recovery was determined when clinical symptoms and signs of peptic ulcer, including the mucosal defect, were no longer present, as determined by gastroscopy; ii) partially effective treatment was determined in cases where clinical symptoms and signs were markedly decreased, but not absent, and $>50 \%$ the mucosal defect area had been repaired; iii) ineffective treatment was determined in cases where the clinical symptoms and signs were increased or unchanged, and the mucosal defect was not filled or was enlarged. In addition, a negative result for $H$. pylori as determined by gastroscopic biopsies, or a $\mathrm{CO}_{2}$ concentration $<100 \mathrm{dpm} / \mathrm{mM}$ as determined by a $\mathrm{Hp}\left[{ }^{14} \mathrm{C}\right]$-urea breath test indicated the absence of $H$. pylori infection.

Isolation and culture of circulating EPCs. Peripheral blood samples $(20 \mathrm{ml})$ were obtained from all three groups; these were drawn from the peptic ulcer patients following anti-ulcer treatment for 8 weeks. Of these samples, $15 \mathrm{ml}$ was used for EPC isolation and culture and $5 \mathrm{ml}$ for flow cytometry. Peripheral blood mononuclear cells (PBMCs) were isolated by magnetic bead selection. Briefly, a single-cell suspension $\left(1 \times 10^{8}\right.$ cells/300 $\left.\mu \mathrm{l}\right)$ was prepared by standard methods (12). A total of $100 \mu \mathrm{l}$ beads (Miltenyi Biotec $\mathrm{GmbH}$, Bergisch Gladbach, Germany), CD133 antigen and $100 \mu 1 \mathrm{Fc}$-receptor (Miltenyi Biotec $\mathrm{GmbH}$ ) were added to the cell suspension and incubated for $40 \mathrm{~min}$ at $4^{\circ} \mathrm{C}$. The cells were subsequently placed in an LS Magnetic Cell Sorting column (Miltenyi Biotec $\mathrm{GmbH}$ ) in a magnetic field. The trapped cells were released by placing the column in a Dynal magnet (Invitrogen; Thermo Fisher Scientific, Inc., Waltham, MA, USA) for at least $1 \mathrm{~min}$. $\mathrm{CD}_{133^{+}}$cells isolated from the blood samples were collected in Medium 199 (Sigma-Aldrich, St. Louis, MO, USA) with the following supplements (all from PeproTech, Rocky Hill, NJ, USA): Medium 199, $10 \%$ fetal bovine serum, $10 \mu \mathrm{g} / \mathrm{l}$ vascular endothelial growth factor (VEGF) and $2 \mu \mathrm{g} / \mathrm{l}$ basic fibroblast growth factor.

The cells were seeded into fibronectin-treated 6-well culture plates (PeproTech) at a final density of $1 \times 10^{7}$ cells/well. At $48 \mathrm{~h}$ after seeding, nonadherent cells $\left(1.0 \times 10^{6}\right.$ cells/well $)$ were cultured at $37^{\circ} \mathrm{C}$ in an atmosphere containing $5 \% \mathrm{CO}_{2}$ for 21 days. The culture medium was changed every 2-3 days. During culture, an inverted phase contrast microscope (IX70-81FZ; Olympus Corporation, Tokyo, Japan) was used to observe the EPC morphology and growth in vitro at days 3, 7, 10,14 and 21 of culture.

Identification of vascular endothelial cells. To identify vascular endothelial cells, dual staining for fluorescently labeled Dil-acetylated-low density lipoprotein (Dil-ac-LDL; Molecular Probes; Thermo Fisher Scientific, Inc.) and fluorescein isothiocyanate (FITC)-Ulex europaeus agglutinin (UEA)-1 (Sigma-Aldrich) was performed on day 4 of the culture. The cells were incubated at $37^{\circ} \mathrm{C}$ for $4 \mathrm{~h}$ with $10 \mu \mathrm{g} / \mathrm{ml}$ Dil-ac-LDL, and then fixed with $4 \%$ paraformaldehyde for $10 \mathrm{~min}$. Subsequent to PBS washing, the cells were treated with $10.0 \mu \mathrm{g} / \mathrm{ml}$ FITC-UEA-1 for $30 \mathrm{~min}$. A laser scanning confocal microscope (TCS-SP5; Leica Microsystems GmbH, Wetzlar, Germany) was used for observation, differentiation and identification.

Flow cytometry of circulating EPCs. A 5-ml blood sample (from the aforementioned $20 \mathrm{ml}$ sample) was used for EPC counting. Approximately $0.2 \mathrm{ml}$ mononuclear cells obtained by magnetic bead selection was used for cell counting. Briefly, $0.2 \mathrm{ml}$ cell suspension was incubated with monoclonal mouse phycoerythrin-conjugated anti-CD34 antibody (dilution, 1:1,000; cat. no. FAB7227P; R\&D Systems, Inc., Minneapolis, MN, USA), mouse FITC-conjugated anti-CD45 (dilution, 1:2,000; cat. no. 9625-02; SouthernBiotech, Birmingham, AL, USA) and monoclonal mouse phycoerythrin-conjugated anti-type 2 VEGF (VEGF-R2; dilution, 1:1,000; cat. no. FAB357P; R\&D Systems, Inc.) at room temperature for $20 \mathrm{~min}$ in the dark. The 
cells were then blocked for non-specific binding by incubation in red blood cell lysate for $15 \mathrm{~min}$. Samples with a density of $1 \times 10^{6}$ cells were analyzed using a FC5000 cytometer (Beckman Coulter, Inc., Brea, CA, USA).

Circulating EPCs were negative for the leucocyte marker CD45, positive for the prototypical stem cell marker CD34, and positive for the endothelial cell marker VEGF-R2 (13).

Circulating EPC colony counts. Colonies were evaluated after 7 days of culture, and a colony was defined as a central core of 'round' cells with elongated 'sprouting' cells at the periphery. Three researchers independently counted the EPC colonies, and experiments were conducted four times per patient.

Statistics analysis. Values are presented as the mean \pm standard deviation. The statistical significance of the data was first assessed using a Kolmogorov-Smirnov test. Comparison of contingency values and frequency was analyzed using a $\chi^{2}$ test. Multiple comparisons were performed with a one-way analysis of variance, and Student's t test was applied for single comparisons. Statistical analyses were conducted using SPSS version 12 (SPSS, Inc., Chicago, Il, USA). P<0.05 was considered to indicate a statistically significant result.

\section{Results}

Treatment efficacy in the experimental groups. The treatment efficacy in the experimental groups was evaluated (Table I). Treatment was partially effective in 12 of the 32 peptic ulcer patients with T2DM, and ineffective in the remaining 20 patients. Notably, 25 patients were defined as completely recovered following treatment, and the treatment was considered partially effective in 7 patients among the 32 peptic ulcer patients without T2DM. The treatment was therefore more effective in patients without T2DM than those with T2DM.

EPC characterization. EPC characterization was conducted using fluorescence microscopy. PBMCs were found to exhibit circular morphology and were suspended in the medium. The volume of adherent cells increased following 3 days of culture, as well as the number of diamond-shaped cells. Colonies of adherent cells began to appear at day 7. The majority of the cells were of bipolar spindle shape and exhibited a cable-like structure at day 14. As shown in Fig. 1, the adherent cells displayed cobble stone-like morphology at day 21 . Notably, flow cytometry immunophenotyping revealed that isolated cells expressed Dil-ac-LDL and UEA-1 after 10 days of culturing (Fig. 2).

Comparison of changes in EPCs in the three treatment groups. To evaluate the number of vascular endothelial cells derived from circulating EPCs in the three groups, cells were counted using flow cytometry. As shown in Fig. 3, group C (healthy control patients) exhibited the highest circulating EPC-forming ability with $1,045 \pm 106$ cells $/ \mathrm{ml}$, whereas group A (peptic ulcer patients with T2DM) exhibited the lowest ability with $532 \pm 90$ cells $/ \mathrm{ml}$. The number of circulating EPCs in group A was significantly decreased compared with groups B (peptic ulcer patients without T2DM; 1,002 \pm 93 cells $/ \mathrm{ml}$ ) and $\mathrm{C}(\mathrm{P}<0.05)$, while no significant difference was observed between groups $\mathrm{B}$ and $\mathrm{C}(\mathrm{P}>0.05)$.

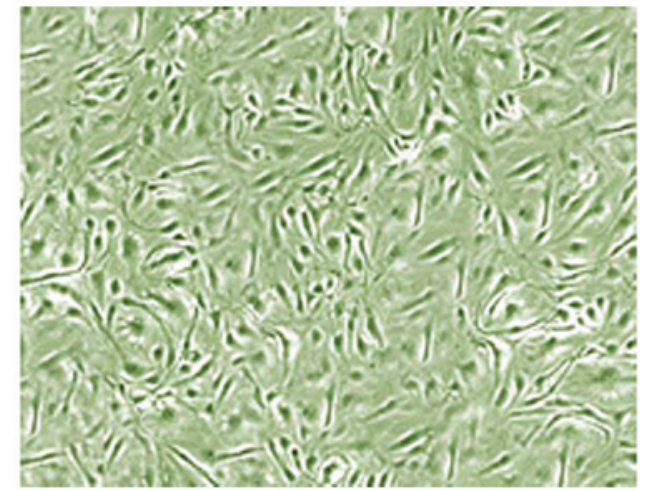

Figure 1. Phase contrast image showing endothelial progenitor cell morphology at day 21 of culture. Magnification, x200.

A

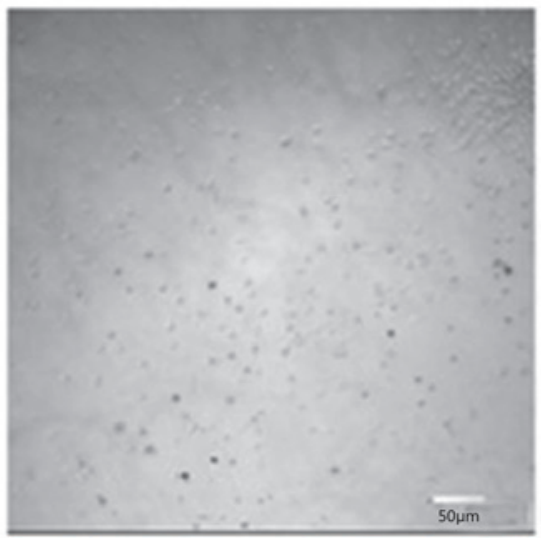

B

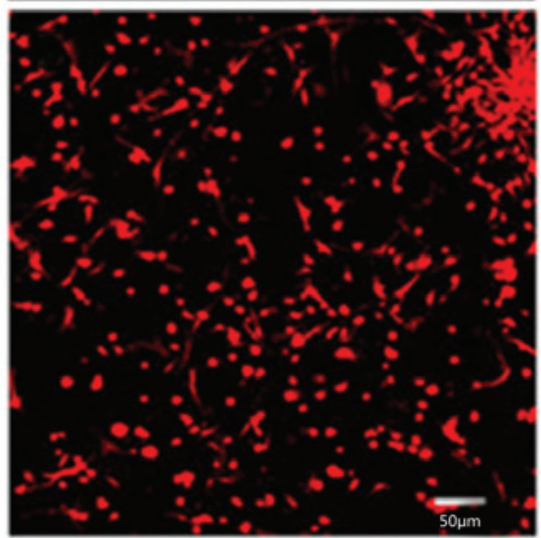

C

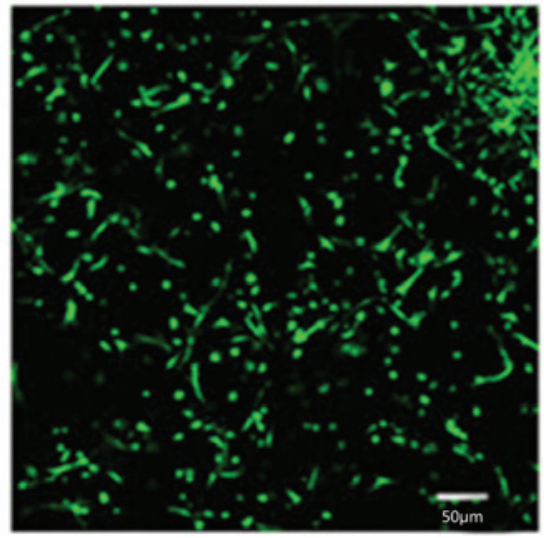

Figure 2. Fluorescence detection of $\mathrm{CD} 133^{+}$mononuclear cells; images are from a representative control cell population. Magnification, x100. (A) Phase contrast images; (B) Dil-acetylated-low density lipoprotein fluorescence image; (C) fluorescein isothiocyanate-Ulex europaeus agglutinin-1 fluorescence image. 
Table I. Treatment effect for peptic ulcer patients.

Peptic ulcer patients with T2DM $(\mathrm{n}=32)$

Category

Partially effective

Peptic ulcer patients without T2DM (n=32)

Complete recovery Partially effective

No. of patients, $n$

Male/female patients, $\mathrm{n} / \mathrm{n}$

$\begin{array}{cc}12 & 20 \\ 8 / 4 & 10 / 10 \\ 63.9 \pm 5.2 & 64.3 \pm 5.4\end{array}$

25

$15 / 10$ 7

$2 / 5$

Age, years

$63.9 \pm 5.2$

$64.3 \pm 5.4$

$65.3 \pm 3.8$

$64.6 \pm 5.5$

T2D2M, type 2 diabetes mellitus.
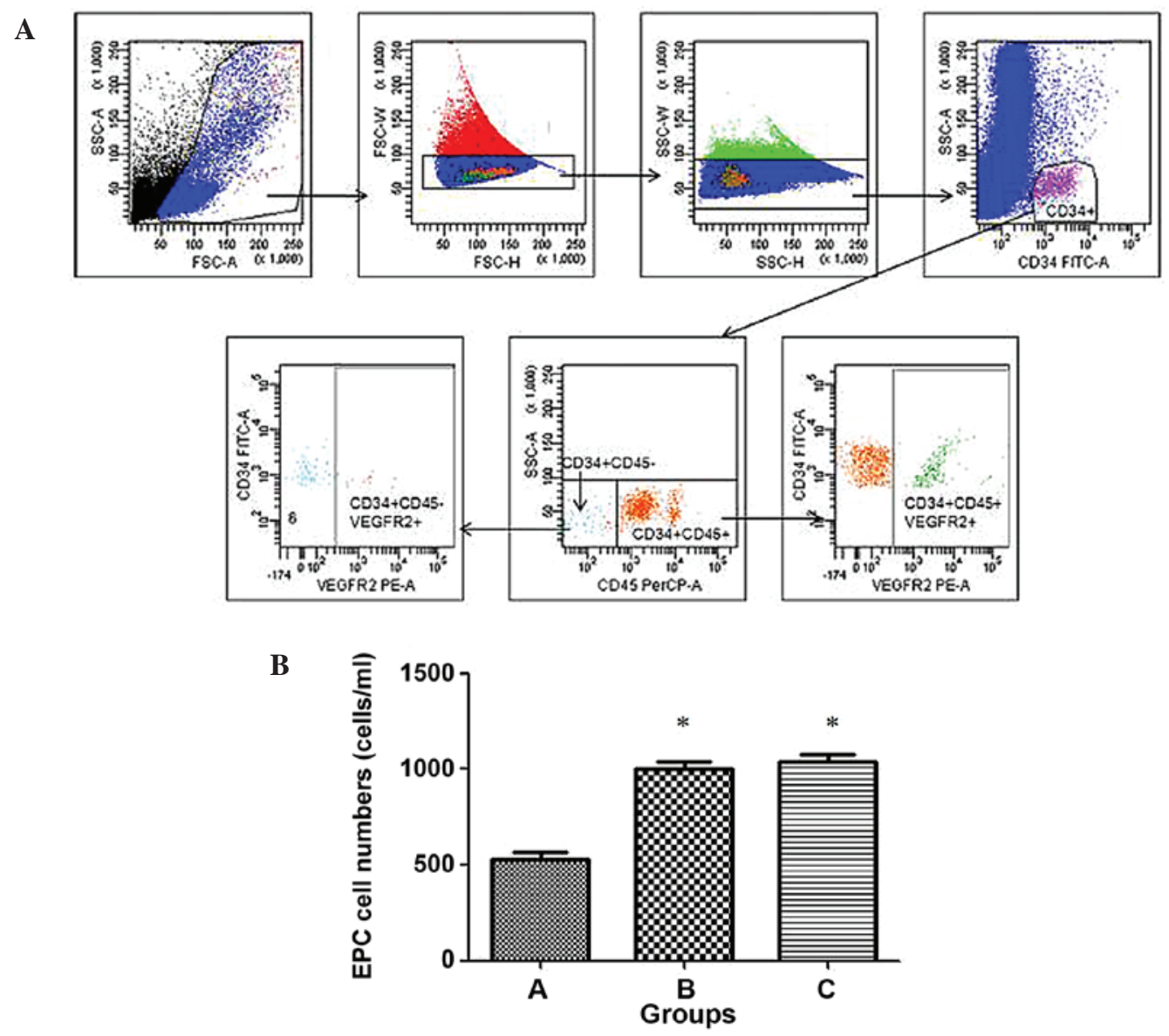

Figure 3. (A) Flow cytometric analysis and number of circulating EPCs in the three groups sorted according to the presence of CD34, CD45, or VEGFR-R2. (B) Changes in EPC measurements in peptic ulcer patients with T2DM (group A), peptic ulcer patients without T2DM (group B), and healthy controls (group C). ${ }^{*} \mathrm{P}<0.05$, vs. group A. EPC, endothelial progenitor cells; T2DM, type 2 diabetes mellitus.

A

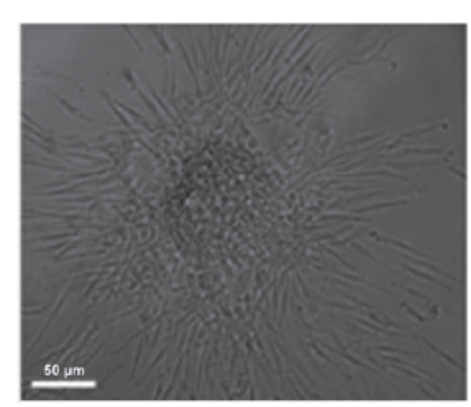

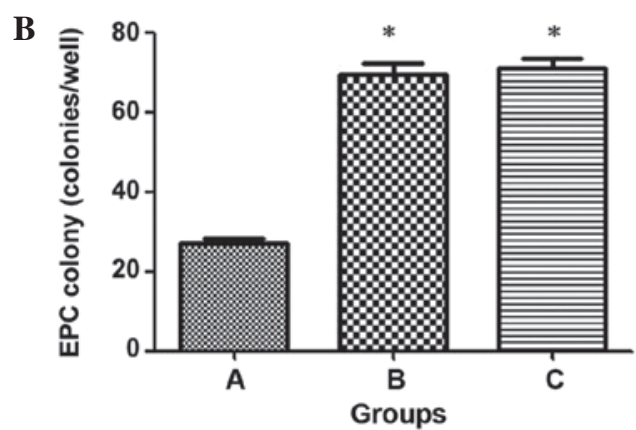

Figure 4. Colonies of circulating EPCs and columnar analysis of EPC colony formation. (A) A colony of circulating EPCs is shown, as well as (B) changes in circulating EPC colony formation in peptic ulcer patients with T2DM (group A), peptic ulcer patients without T2DM (group B) and healthy controls (group C). ${ }^{*} \mathrm{P}<0.05$ vs. group A. EPC, endothelial progenitor cells; T2DM, type 2 diabetes mellitus. 
To evaluate the colony forming ability of the circulating EPCs in the three groups, colonies were counted by three independent researchers (Fig. 4). Similarly, group C exhibited the highest circulating EPC forming ability $(70 \pm 9$ colonies $/ \mathrm{ml})$, whereas group A exhibited the lowest ability $(28 \pm 5$ colonies $/ \mathrm{ml}$ ). The number of circulating EPC colonies in group A were significantly increased compared with groups B $(68 \pm 8$ colonies $/ \mathrm{ml})$ and $\mathrm{C}(\mathrm{P}<0.05)$, whereas group $\mathrm{B}$ did not reveal significantly different circulating colony numbers to group C (Fig. 4) ( $\mathrm{P}>0.05)$. This indicates that the EPC forming ability may be significantly disrupted in group A.

\section{Discussion}

A previous study demonstrated that circulating EPCs were associated with microvascular disease (7), whereas studies have yet to report the existence of an association between circulating EPCs and ulcer treatment. The results of the present study indicated that, compared with peptic ulcer patients without T2DM, peptic ulcer patients with T2DM exhibited poor treatment outcomes. In addition, the number of EPCs was most significantly decreased in peptic ulcer patients with T2DM, compared with the other two groups. Furthermore, the lowest colony forming ability of circulating EPCs was present in peptic ulcer patients with T2DM.

The present study measured the most currently used phenotypic markers for assessing vascular endothelial cells, including CD34, CD133 and VEGR-R2 (14). The results indicated that peptic ulcer patients with T2DM had significantly reduced numbers of circulating EPCs, compared with peptic ulcer patients without T2DM and healthy controls. Furthermore, following treatment with peptic ulcer drugs, patients with and without T2DM exhibited significantly reduced colony forming abilities, as compared with healthy controls. It is widely known that angiogenesis and tissue repair are required for ulcer healing (15). In addition, previous studies demonstrated that EPC injury reduces regeneration, contributing to low microvascular density, slow blood vessel formation and delayed cellular renewal, suggesting an important role for EPCs in endogenous vascular repair $(16,17)$. The results of the present study suggested that ulcer treatment may be associated with EPC impairment.

Patients with diseases associated with T2DM were demonstrated to have lower levels of circulating EPCs $(18,19)$, results which were not concordant with a hypothesis of association between ulcer treatment and EPC injury. However, the lower colony-forming ability of circulating EPCs from peptic ulcer patients compared with that in healthy controls suggested a correlation between EPC injury and peptic ulcers. Further studies that examine the association between EPCs and ulcer treatment, and adjust to EPC changes induced by T2DM are therefore required.

Recent studies have sought stem cell-based approaches to harness vascular regeneration in view of its capacity for self-renewal and directed differentiation (20-22). Given the hypothetical ability of EPCs to differentiate and form new blood vessels, the use of EPCs for vascular regeneration was presented by Asahara et al (23) in 1997. Recently, stem cell-based therapy has been studied in clinical trials $(24,25)$. The present study demonstrated the role of EPCs in ulcer treatment, and we therefore propose that EPC therapy may also be used in peptic ulcer-associated diseases.

In conclusion, the results of the present investigation indicated that ulcer treatment is associated with reduction in circulating EPC number. In addition, the ability of circulating EPCs to differentiate into vascular endothelial cells was lowest in peptic ulcer patients with T2DM, suggesting the important role of circulating EPCs in ulcer treatment. However, further studies are required in order to examine the association between EPCs and ulcer treatment following adjustment to the effect of T2DM on circulating EPCs.

\section{Acknowledgements}

The present study was supported by grants from the Key Discipline Construction Project of the Pudong Health Bureau of Shanghai (no. PWZx2014-03), the General Program of Shanghai Municipal Health Bureau (no. 2007094) and the Subject Leader Plan of Pudong Health Bureau of Shanghai (no. pWRd2014-02). The authors would like to thank colleagues in the gastroscopy, laboratory and endocrinology departments for their assistance with this study.

\section{References}

1. Tacheci I and Bures J: Peptic ulcer disease in patients with diabetes mellitus. Vnitr Lek 57: 347-350, 2011 (In Czech).

2. Najm WI: Peptic ulcer disease. Prim Care 38: 383-394, 2011.

3. GBD 2013 Mortality and Causes of Death Collaborators: Global, regional, and national age-sex specific all-cause and cause-specific mortality for 240 causes of death, 1990-2013: A systematic analysis for the Global Burden of Disease Study 2013. Lancet 385: 117-171, 2015.

4. Dey L, Attele AS and Yuan CS: Alternative therapies for type 2 diabetes. Altern Med Rev 7: 45-58, 2002.

5. Lorenzi M: Glucose toxicity in the vascular complications of diabetes: The cellular perspective. Diabetes Metab Rev 8: 85-103, 1992.

6. Urbich $\mathrm{C}$ and Dimmeler S: Endothelial progenitor cells: Characterization and role in vascular biology. Circ Res 95: 343-353, 2004.

7. Werner N, Kosiol S, Schiegl T, Ahlers P, Walenta K, Link A, Böhm M and Nickenig G: Circulating endothelial progenitor cells and cardiovascular outcomes. N Engl J Med 353: 999-1007, 2005.

8. Fadini GP, Miorin M, Facco M, Bonamico S, Baesso I, Grego F, Menegolo M, de Kreutzenberg SV, Tiengo A, Agostini C and Avogaro A: Circulating endothelial progenitor cells are reduced in peripheral vascular complications of type 2 diabetes mellitus. J Am Coll Cardiol 45: 1449-1457, 2005.

9. Tecilazich F, Dinh T, Pradhan-Nabzdyk L, Leal E, Tellechea A, Kafanas A, Gnardellis C, Magargee ML,Dejam A, Toxavidis V, et al: Role of endothelial progenitor cells and inflammatory cytokines in healing of diabetic foot ulcers. PLoS One 8: e83314, 2013.

10. Alberti KG and Zimmet PZ: Definition, diagnosis and classification of diabetes mellitus and its complications. Part 1: diagnosis and classification of diabetes mellitus provisional report of a WHO consultation. Diabet Med 15: 539-553, 1998.

11. Editorial Board of Chinese Journal of Digestion: Suggestion on standardized diagnosis and treatment of peptic ulcer disease (2008, Huang Shah). Zhong Hua Xiao Hua Za Zhi 28: 447-450, 2008 (In Chinese).

12. Asahara T, Murohara T, Sullivan A, Silver M, van der Zee R, Li T, Witzenbichler B, Schatteman G and Isner JM: Isolation of putative progenitor endothelial cells for angiogenesis. Science 275: 964-967, 1997.

13. Hirschi KK, Ingram DA and Yoder MC: Assessing identity, phenotype and fate of endothelial progenitor cells. Arterioscler Thromb Vasc Biol 28: 1584-1595, 2008.

14. Bertolini F, Mancuso P, Braidotti P, Shaked Y and Kerbel RS: The multiple personality disorder phenotype(s) of circulating endothelial cells in cancer. Biochim Biophys Acta 1796: 27-32, 2009. 
15. Tarnawski AS: Cellular and molecular mechanisms of gastrointestinal ulcer healing. Dig Dis Sci 50 (Suppl 1): S24-S33, 2005.

16. Schmidt-Lucke C, Rössig L, Fichtlscherer S, Vasa M, Britten M, Kämper U, Dimmeler S and Zeiher AM: Reduced number of circulating endothelial progenitor cells predicts future cardiovascular events: Proof of concept for the clinical importance of endogenous vascular repair. Circulation 111: 2981-2987, 2005.

17. Hristov M, Erl W and Weber PC: Endothelial progenitor cells: Mobilization, differentiation and homing. Arterioscler Thromb Vasc Biol 23: 1185-1189, 2003.

18. Brunner S, Hoellerl F, Schmid-Kubista KE, Zeiler F, Schernthaner G, Binder S and Schernthaner GH: Circulating angiopoietic cells and diabetic retinopathy in type 2 diabetes mellitus, with or without macrovascular disease. Invest Ophthalmol Vis Sci 52: 4655-4662, 2011

19. Li M, Ho JC, Lai KW, Au KK, Xu A, Cheung BM, Lam KS and Tse HF: The decrement in circulating endothelial progenitor cells (EPCs) in type 2 diabetes is independent of the severity of the hypoadiponectemia. Diabetes Metab Res Rev 27: 185-194, 2011.
20. Leeper NJ, Hunter AL and Cooke JP: Stem cell therapy for vascular regeneration: Adult, embryonic, and induced pluripotent stem cells. Circulation 122: 517-526, 2010

21. Tang YL, Zhao Q, Qin X, Shen L, Cheng L, Ge J and Phillips MI Paracrine action enhances the effects of autologous mesenchymal stem cell transplantation on vascular regeneration in rat model of myocardial infarction. Ann Thorac Surg 80: 229-237, 2005.

22. Rafii S and Lyden D: Therapeutic stem and progenitor cell transplantation for organ vascularization and regeneration. Nat Med 9: 702-712, 2003.

23. Asahara T, Murohara T, Sullivan A, Silver M, van der Zee R, Li T, Witzenbichler B, Schatteman G and Isner JM: Isolation of putative progenitor endothelial cells for angiogenesis. Science 275: 964-967, 1997

24. Sen S, McDonald SP, Coates PT and Bonder CS: Endothelial progenitor cells: Novel biomarker and promising cell therapy for cardiovascular disease. Clin Sci (Lond) 120: 263-283, 2011.

25. Zhao YH, Yuan B, Chen J, Feng DH, Zhao B, Qin C and Chen YF: Endothelial progenitor cells: Therapeutic perspective for ischemic stroke. CNS Neurosci Ther 19: 67-75, 2013. 